\title{
Pancreatic Injury Successfully Treated with Endoscopic Stenting for Major Pancreatic Duct Disruption
}

\author{
Takashi Abe ${ }^{1}$, Takayuki Nagai ${ }^{2}$, Kazunari Murakami ${ }^{1}$, Juro Anan ${ }^{1}$, Masahiro Uchida ${ }^{1}$, \\ Hideki Ono ${ }^{1}$, Hitoshi Okawara ${ }^{2}$, Jin Tanahashi ${ }^{1}$, Tadayoshi Okimoto ${ }^{1}$, \\ Masaaki Kodama ${ }^{1}$ and Toshio Fujioka ${ }^{1}$
}

\begin{abstract}
We present a 43-year-old Japanese man with major pancreatic duct disruption caused by blunt pancreatic head damage. Computed tomography (CT) revealed pancreatic head injury, and endoscopic retrograde pancreatography showed pancreatic duct disruption at the injury site along with contrast media leakage. We placed a pancreatic stent for 3 months, after which closure of the pancreatic duct fistula was confirmed. CT on the 9th hospital day showed acute pancreatic fluid collections, but these had disappeared at the 3 month follow-up CT. The patient has remained asymptomatic at follow-up for 3 years.
\end{abstract}

Key words: pancreatic injury, major pancreatic duct disruption, endoscopic stenting

(Inter Med 48: 1889-1892, 2009)

(DOI: 10.2169/internalmedicine.48.2331)

\section{Introduction}

Although nonoperative management of minor pancreatic injury is widely accepted, the treatment of ductal disruption remains debated. Traditionally, pancreatic injury with major pancreatic duct (MPD) disruption has been treated surgically. Here, we report a case of pancreatic injury with MPD disruption that was successfully treated by pancreatic stenting.

\section{Case Report}

A 43-year-old Japanese man was admitted to our hospital for upper abdominal pain in January 2006, 12 hours after receiving a blunt abdominal injury from an iron frame at his job as a construction worker. His vital signs were stable. Laboratory studies revealed a white blood cell (WBC) count of $10,800 / \mu \mathrm{L}$ (normal range, 3,400-9,400/ $\mu \mathrm{L}$ ), serum amylase (AMY) $795 \mathrm{IU} / \mathrm{L}$ (normal range 60-170 IU/L), serum amylase isozyme $77.4 \%$ (normal range 13-47\%) and Creactive protein (CRP) $0.34 \mathrm{mg} / \mathrm{dL}$ (normal range $<0.30 \mathrm{mg}$ /
dL). Computed tomography (CT) demonstrated partial disruption of the parenchyma with a small amount of peripancreatic fluid at the pancreatic head (Fig. 1A). Endoscopic retrograde pancreatography (ERP) on the 2nd hospital day showed leakage of contrast from a disrupted MPD; the contrast was confined to the pancreatic parenchyma without retroperitoneal spread at the injury site (Fig. 2A). We therefore diagnosed pancreatic head injury with MPD disruption (grade IV according to the American Association for the Surgery of Trauma (AAST) Injury Scale II (1)). Upper endoscopic examination showed erosions at the duodenal bulb, but there was no other serious multiple organ injury. We placed a 5-French endoscopic nasobiliary drainage (ENBD) tube (Flexima, Boston Scientific, Boston, MA, USA) to cross the injured site (Fig. 2B). On the 3rd day, the ENBD tube slipped from the pancreatic duct, and the laboratory studies showed deterioration: WBC 22,780/ $\mathrm{LL}$, AMY 957 IU/L, and CRP $19.82 \mathrm{mg} / \mathrm{dL}$. We therefore replaced the ENBD tube with a 7-French, 5-cm endoscopic pancreatic stent (EPS) (Geenen type, Wilson-Cook Medical, WinstonSalem, NC, USA) (Fig. 2C). On the 9th day, laboratory parameters were improved (WBC 6,990/ $\mathrm{L}$, AMY 327 IU/L,

${ }^{1}$ Department of General Medicine and Gastroenterology, Faculty of Medicine, Oita University, Yufu and ${ }^{2}$ Department of Gastroenterology, Oita Kouseiren Tsurumi Hospital, Beppu

Received for publication April 4, 2009; Accepted for publication July 22, 2009

Correspondence to Dr. Takashi Abe, takashi0315@med.oita-u.ac.jp 

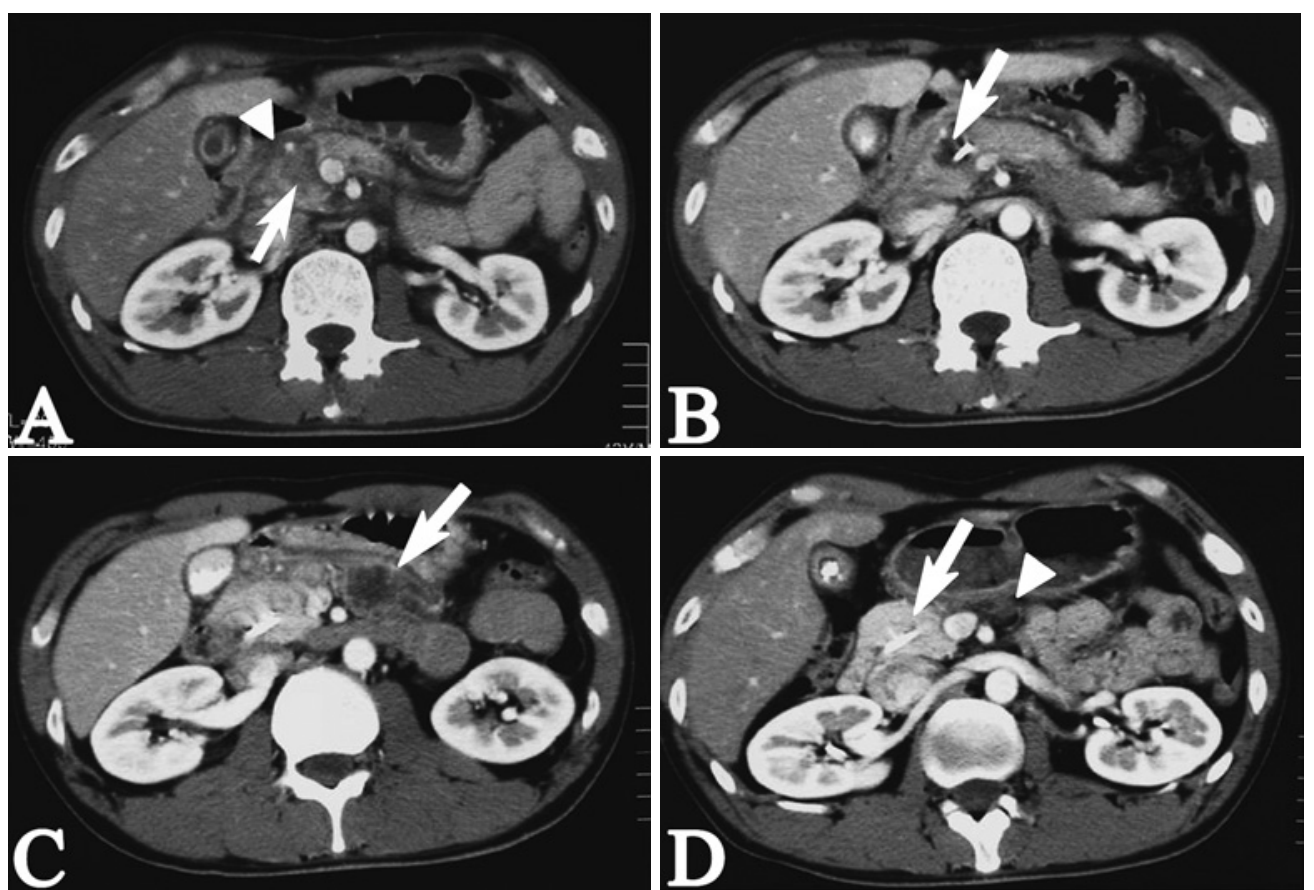

Figure 1. (A) Initial computed tomography (CT) suggested a partial disruption of the pancreatic parenchyma (arrow) and a small amount of fluid around the pancreatic gland (arrow head) at the pancreatic head. (B) CT on the 9th hospital day showed the appearance of a pseudocyst at the pancreatic head (arrow). (C) CT on the 9th day showed a pseudocyst in the pancreatic body (arrow). (D) CT at 3 months showed disappearance of the pseudocysts at the pancreatic head (arrow) and body (arrow head).
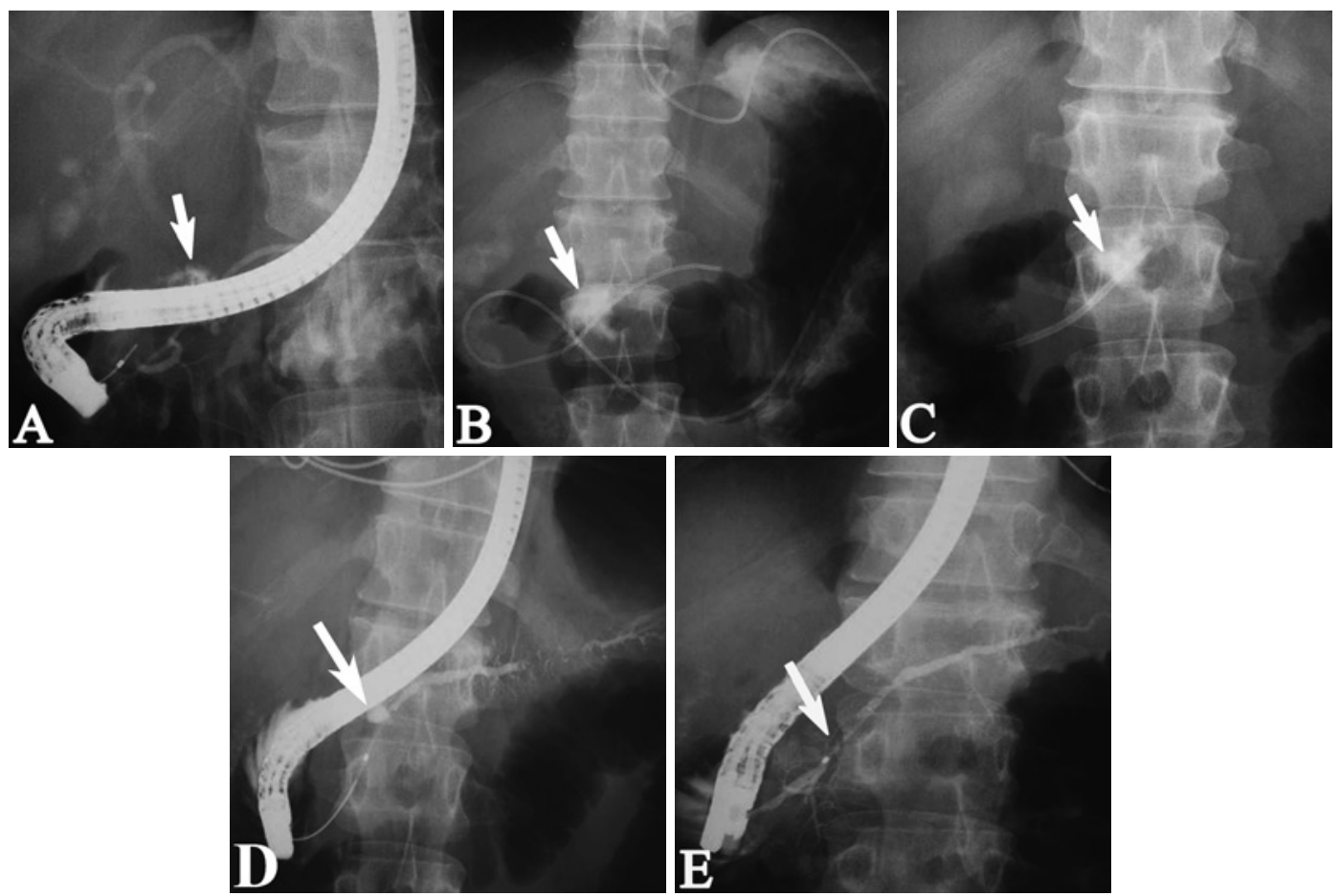

Figure 2. (A) Initial endoscopic retrograde pancreatography (ERP) showed leakage of contrast material localized to the pancreatic parenchyma (arrow). (B) An endoscopic nasobiliary drainage (ENBD) tube (5 French) was placed to cross the injured site at the initial ERP (arrow). (C) An endoscopic pancreatic stent (EPS, 7 French, $5 \mathrm{~cm}$ ) was placed to cross the injured site on the 3rd day (arrow). (D) ERP on the 30th day showed that the leakage of contrast remained, and revealed a mild stricture at the injury site (arrow). (E) ERP at 3 months showed disappearance of the leakage, but the mild stricture remained at the injured site (arrow) without secondary upstream ductal dilation. 


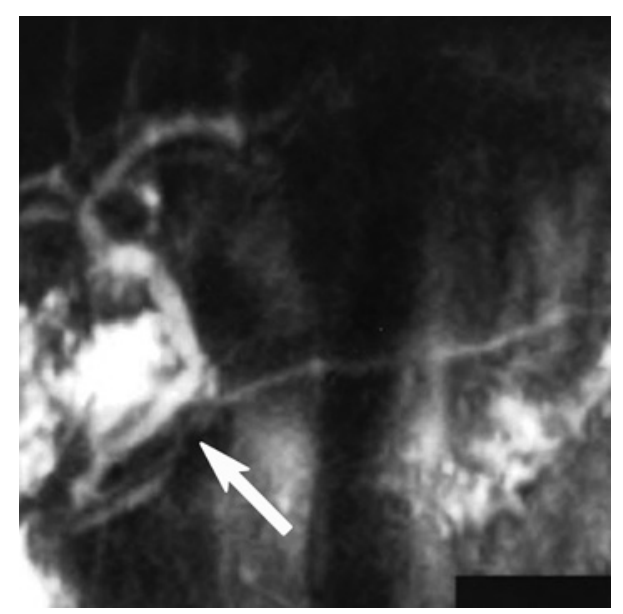

Figure 3. Magnetic resonance cholangiopancreatography (MRCP) at 1 year and 3 months showed that the mild stricture of the major pancreatic duct (MPD) remained at the injured site (arrow) without secondary upstream ductal dilation.

and CRP $1.21 \mathrm{mg} / \mathrm{dL}$ ) and his abdominal pain was relieved, but follow-up CT revealed acute fluid collections at the pancreatic head and body (Fig. 1B, Fig. 1C). On the 18th day, follow-up ERP showed that leakage of contrast remained, and the EPS was replaced with one that was 5 French and 7 $\mathrm{cm}$. The longer stent was chosen to ensure that it crossed the site of the injured duct. On the 19th day, a low-fat diet was resumed. On the 30th day, follow-up ERP showed that leakage of contrast from a disrupted MPD remained, and revealed mild stricture at the injury site (Fig. 2D), and a 7French, 7-cm EPS was placed for further dilation. He was discharged on the 35th day, without abdominal symptoms. After 3 months, follow-up CT revealed disappearance of the pancreatic fluid collection (Fig. 1D), and follow-up ERP showed that leakage of contrast had disappeared but the mild stricture remained without secondary upstream ductal dilation (Fig. 2E), so the EPS was removed. At 1 year and 3 months, magnetic resonance cholangiopancreatography (MRCP) showed that the mild stricture remained unchanged (Fig. 3), but CT was almost normal. The patient remained has asymptomatic for the 3 years of follow-up.

\section{Discussion}

Pancreatic injury is relatively rare, occurring in only 3 to $12 \%$ of all patients with severe abdominal trauma (2). The diagnosis is often delayed because of the absence of specific symptoms, the unusual nature of physical findings, and nonspecific laboratory findings. CT is a simple, noninvasive method for the initial assessment of traumatic pancreatic injury (3). The condition of the MPD is the determining factor in management of pancreatic injury; this should therefore be evaluated post-trauma when ductal injury is suspected. When abdominal CT shows a large hematoma or deep laceration of the pancreatic parenchyma, MPD injury should be suspected (4). ERP is one of the most useful methods for demonstrating MPD injury (3,5-10) and should be performed as soon as possible (4). However, as ERP is an invasive procedure, it is not suitable when the patient requires emergency laparotomy $(3,4)$.

Delayed diagnosis of MPD injury increases mortality and morbidity (11-13), and therefore surgical treatment has traditionally been thought to be the one intervention that is mandatory in MPD injury. However, inadequate resection or undetected duct injury may lead to various postoperative complications including pancreatic fistula, abscess, pseudocyst, bleeding, and persistent pancreatic pain (3). Recently, some case series have shown pancreatic duct stent placement to be an effective therapy in resolving pancreatic duct disruption and its sequelae $(3,4,10,14)$. Kim et al (3) claim that insertion of an endoprosthesis is an effective therapeutic modality in patients with MPD injury in whom contrast leakage is confined to the pancreatic parenchyma.

Lin et al (4) reported long-term results of stent placement in traumatic pancreatic duct disruption. They chose 5-French stents for initial placement, changing to 7-French for further dilation. The choice of stent length depends on the site of injury, and it is important to place the stent through the leak when possible. In the pancreatic head, a 7-cm stent is adequate, and 9,12 , or $15 \mathrm{~cm}$ stents can be used for the body and tail.

Ductal stricture at the injured site is a major complication in the long term, and once a ductal stricture is formed, it is treated with repeated stenting, which may cause epithelial injury and more severe stricture. Given the inevitability of ductal stricture and the need for prolonged, repeated ERP stent exchange, the procedure may not suitable for acute MPD injuries involving the pancreatic body and tail (AAST grade III) (4). Lin et al. (4) reported that stent placement can avoid surgery in the acute stage and may be reserved as another choice for acute AAST grade IV pancreatic injury. But they reported that one patient in their series developed sepsis and died, and they concluded that pancreatic duct stent placement is not risk free, and that further studies are therefore needed to define the usefulness of pancreatic duct stenting in the treatment of acute blunt pancreatic injury. Chronic renal failure may be a risk factor, and contrast medium leaking into the retroperitoneal space is another risk factor (4). When contrast medium leak into the retroperitoneal space or even into the peritoneal cavity, the injury is more serious and operation is recommended (15).

In the present case, MPD injury was shown on ERP, and pancreatic stent replacement was performed soon after blunt abdominal trauma. This procedure achieved rapid clinical improvement and enabled surgery to be avoided. On the 3rd day, we replaced the ENBD tube with a 5-cm EPS, but it just crossed the site of the injured duct. If a longer EPS had been used as the replacement on the 3rd day, the acute pancreatic fluid collection at the pancreatic body may have been prevented by better facilitation of pancreatic juice drainage.

In conclusion, pancreatic duct stenting in acute AAST grade IV MPD disruption may avoid surgery and be a useful 
procedure when this injury is caused by blunt abdominal trauma. Pancreatic stenting should be reserved as another choice therapeutic modality in such cases before any definitive surgical attempt. Larger controlled studies are needed to confirm whether or not stent-placement improves post-ERP morbidity and mortality.

\section{Acknowledgement}

The authors thank Mr. Kai (Oita Kouseiren Tsurumi Hospital) for his excellent technical assistance.

\section{References}

1. Moore EE, Cogbill TH, Malangoni MA, et al. Organ injury scaling II: Pancreas, duodenum, small bowel, colon, and rectum. J Trauma 30: 1427-1429, 1990.

2. Jurkovich GJ, Carrico CJ. Pancreatic trauma. Surg Clin North Am 70: 575-593, 1990 .

3. Kim HS, Lee DK, Kim IW, et al. The role of endoscopic retrograde pancreatography in the treatment of traumatic pancreatic duct injury. Gastrointest Endosc 54: 49-55, 2001.

4. Lin BC, Liu NJ, Fang JF, Kao YC. Long-term results of endoscopic stent in management of blunt major pancreatic duct injury. Surg Endosc 20: 1551-1555, 2006.

5. Doctor N, Dooley JS, Davidson BR. Assessment of pancreatic damage following trauma: is endoscopic retrograde cholangiopancreatography the gold standard? Postgrad Med J 71: 116-117, 1995

6. Stone A, Sugawa C, Lucas C, Hayward S, Nakamura R. The role of endoscopic retrograde pancreatography (ERP) in blunt abdominal trauma. Am Surg 56: 715-720, 1990.

7. Barkin JS, Ferstenberg RM, Panullo W, Manten HD, Davis RC Jr. Endoscopic retrograde cholangiopancreatography in pancreatic trauma. Gastrointest Endosc 34: 102-105, 1988.

8. Sugawa C, Lucas CE. The case for preoperative and intraoperative
ERCP in pancreatic trauma. Gastrointest Endosc 34: 145-147, 1988.

9. Whittwell AE, Gomez GA, Byers P, Kreis DJ Jr, Manten H, Casillas VJ. Blunt pancreatic trauma: prospective evaluation of early endoscopic retrograde pancreatography. South Med J 82: 586-591, 1989.

10. Bagci S, Tuzun A, Erdil A, Uygun A, Gulsen M, Dagalp K. Endoscopic treatment of pancreatic duct disruption due to blunt abdominal trauma: a case report. Mil Med 172: 548-550, 2007.

11. Jones RC. Management of pancreatic trauma. Am J Surg 150: 698-704, 1985.

12. Bach RD, Frey CE. Diagnosis and treatment of pancreatic trauma. Am J Surg 121: 20-29, 1971.

13. Heitsch RC, Knutson CO, Fulton RE, Jones CE. Delineation of critical factors in the treatment of pancreatic trauma. Surgery $\mathbf{8 0}$ : 523-529, 1976.

14. Canty TG Sr, Weinman D. Treatment of pancreatic duct disruption in children by an endoscopically placed stent. J Pediatr Surg 36: 345-348, 2001.

15. Takishima T, Hirata M, Kataoka Y, et al. Pancreatographic classification of pancreatic ductal injuries caused by blunt injury to the pancreas. J Trauma 48: 745-752, 2000.

(C) 2009 The Japanese Society of Internal Medicine http://www.naika.or.jp/imindex.html 